\title{
Julien LARREGUE, Héréditaire. L'éternel retour des théories biologiques du crime
}

Jean-Marie Renouard

\section{(2) OpenEdition}

1 Journals

Édition électronique

URL : https://journals.openedition.org/ress/7270

DOI : $10.4000 /$ ress. 7270

ISBN : $1663-4446$

ISSN : $1663-4446$

Éditeur

Librairie Droz

Édition imprimée

Date de publication : 21 mai 2021

Pagination : 264-266

ISSN : 0048-8046

\section{Référence électronique}

Jean-Marie Renouard, « Julien LARREGUE, Héréditaire. L'éternel retour des théories biologiques du crime », Revue européenne des sciences sociales [En ligne], 59-1 | 2021, mis en ligne le 21 mai 2021, consulté le 25 mai 2021. URL : http://journals.openedition.org/ress/7270 ; DOI : https://doi.org/ $10.4000 /$ ress. 7270

Ce document a été généré automatiquement le 25 mai 2021.

(c) Librairie Droz 


\title{
Julien LARREGUE, Héréditaire. L'éternel retour des théories biologiques du crime
}

\author{
Jean-Marie Renouard
}

\section{RÉFÉRENCE}

Julien LARREGUE, 2020, Héréditaire. L'éternel retour des théories biologiques du crime, Paris, Éditions du Seuil, $272 \mathrm{p}$.

1 Depuis les années 1930, la criminologie américaine est dominée par la sociologie. Les sociologues les plus réputés et leurs meilleurs doctorants ont investi l'étude de la criminalité et de la déviance au point que la criminologie américaine, la plus développée de toutes, est la référence partout ailleurs. Les sociologues raflèrent les prix, la présidence des associations et la direction des revues de criminologie, les budgets de recherche et les postes dans les universités les plus prestigieuses. Ils accumulèrent ainsi du capital académique et scientifique. Face à cette domination sans partage, Julien Larregue, en sept chapitres allant de l'autonomisation de la criminologie et de la percée de la criminologie biosociale (les trois premiers chapitres) à son obsolescence (le dernier), pose la question suivante: comment la criminologie biosociale à la recherche de l'influence des facteurs génétiques, neurologiques, physiologiques, familiaux et environnementaux sur le passage à l'acte criminel et les comportements déviants, tente-t-elle depuis les années 1960 de contester la domination des sociologues pour faire reconnaître sa légitimité et pourquoi, malgré ses efforts, elle reste marginale en dépit d'une visibilité plus assurée depuis 2000 ? À cet égard, son livre, issu de sa thèse de doctorat, est fort intéressant. Il invite le lecteur à suivre sa longue enquête, bien construite chronologiquement et bien documentée, sur le fonctionnement du champ criminologique américain, mobilisant un corpus de 188 articles de criminologie biosociale et 22 entretiens avec des criminologues biosociaux et quelques-uns de leurs adversaires. Au niveau théorique, il s'appuie sur la théorie des 
champs de Pierre Bourdieu et sur les travaux en sociologie des sciences en adoptant la plupart du temps de son enquête ce ton neutre promu par Bruno Latour. Bref, Larregue entend expliquer la lente émergence de la criminologie biosociale dans un champ dominée sans partage par la sociologie.

2 Le premier chapitre décrit la patiente autonomisation de la criminologie au $\mathrm{XX}^{\mathrm{e}}$ siècle qui a profité aux sociologues. Aussi la première génération des criminologues biosociaux des décennies 1960-1990 fut-elle marginalisée, malgré l'intérêt des pouvoirs publics pour une autre approche de la criminalité. Le deuxième chapitre montre que cette première génération de criminologues peina à se faire reconnaître en dépit d'un contexte culturel favorable (publications d'ouvrages sur la biologie humaine et valorisation d'une approche médicale de la criminalité); écrasée qu'elle fut sous la domination de la sociologie qu'elle critiqua autant sur le plan scientifique que politique. En réalité, les avancées théoriques de l'approche génétique furent essentiellement produites à l'extérieur du champ criminologique par des généticiens et des psychologues se méfiant de l'étiquette de «criminologue » et produisant des ressources encore peu mobilisables par les biosociaux. Toutefois, même marginalisée (chap.3), la percée du courant biosocial annonça son développement futur. La production et l'accessibilité des bases de données génétiques ainsi que le développement des facultés de criminologie, où entrèrent les biosociaux, favorisèrent leur installation dans le champ. Cependant, leur recrutement dans des universités peu prestigieuses, tournées vers la professionnalisation davantage que vers la recherche, a nui à leur crédibilité tout en leur accordant de la visibilité. Larregue insiste ensuite sur l'hétérogénéité du courant biosocial opposant les criminologues insistant sur les facteurs génétiques dans l'explication du passage à l'acte pour dévaluer les théories sociologiques, aux criminologues cherchant à les renforcer en utilisant les facteurs génétiques pour mieux éclairer leurs zones d'ombres (chap. 4). En effet, alors que l'on aurait pu s'attendre à ce que leur courant dépasse le débat nature/culture, des controverses à opposent de plus belle les criminologues biosociaux pro-génétique aux pro-environnement (chap.5) La confrontation recherchée avec les sociologues, toujours dominants, permit aux biosociaux, "convaincus que la race est un facteur criminogène" (p. 204) d'être plus souvent cités dans les revues de criminologie et d'acquérir ainsi du capital scientifique en accusant «la sociologie d'être une pseudoscience idéologique » (p. 187). Leur agressivité à l'égard des sociologues fut finalement une stratégie payante. Mais ces derniers " persuadés que leur conception du crime est plus scientifiquement solide, [...] se contentent d'ignorer les provocations de cette minorité bruyante » (chap. 6, p. 205).

3 Dans le dernier chapitre, Larregue s'interroge sur l'obsolescence de la criminologie biosociale. D'une part, elle s'appuie sur les données de seconde main de la génétique peu coûteuses et plus faciles à exploiter que les coûteuses données neurologiques ; d'autre part, ses méthodes se voient menacées par le développement de la génomique. Tandis que les sociologues l'ignorent, les neuroscientifiques la tiennent donc soigneusement à distance. Aussi, est-elle condamnée à répéter le même type de recherches et à accumuler le même type de résultats, un peu comme la criminologie mainstream pourrait-on ajouter.

4 En conclusion, après avoir rappelé que les méthodes mises en œuvre et les corpus utilisés par les biosociaux «n'étaient pas en phase avec les derniers standards de la recherche génétique » (p. 234) et que les résultats de leurs travaux n'ont jamais fait 
consensus parmi les scientifiques, Larregue note que le monde judiciaire y a cependant recours, par exemple au pénal, pour déresponsabiliser l'auteur d'une infraction dont l'examen aurait montré une anomalie ou une défaillance génétique.

5 Le lecteur ne doit pas s'attendre à un examen critique des théories biosociales en les comparant entre elles ou avec d'autres théories mais à une analyse des stratégies des biosociaux pour jouer des coudes dans le champ de la criminologie. Le livre de Larregue relève à cet égard moins de la criminologie que de la sociologie des sciences comme en témoignent ses nombreuses références à cette discipline. On peut cependant douter de la réalité d'un éternel retour des théories biologiques du crime - comme l'annonce le sous-titre de l'ouvrage -, car en définitive elles ont marqué continument, certes avec des hauts et des bas, l'histoire de la criminologie depuis Cesare Lombroso, leur fondateur à la fin du XIX ${ }^{\mathrm{e}}$ siècle. Les progrès des neurosciences depuis quelques décennies ont pour effet que l'utilisation de ces théories s'étend davantage, selon l'auteur, en économie ou en psychologie qu'en criminologie. Sans doute parce que l'ambition des criminologues biosociaux de trouver des gènes communs derrière des comportements punissables aussi différents que le délit d'initié, l'abus de faiblesse, le cambriolage, le non-versement de la pension alimentaire ou le viol à caractère incestueux est évidemment déraisonnable. Il reste qu'on ignore quelles sont leurs motivations, si ce n'est celle d'investir le champ de la criminologie? Ont-ils par exemple un projet politique? À ces questions, Larregue répond avec prudence qu'il s'intéresse aux faits, pas aux intentions.

\section{AUTEURS}

JEAN-MARIE RENOUARD

Paris, CNRS - CESDIP 